\title{
An Assessment of Popular e-Learning Systems via Felder-Silverman Model and a Comprehensive-Learning System using the Tools on Web 2.0
}

\author{
Maria Dominic \\ Department of Computer Applications, Sacred Heart College, India \\ Email:dominic@shctpt.edu \\ Sagayaraj Francis \\ Department of Computer Science and Engineering, Pondicherry Engineering College, India \\ Email: fsfrancis@pec.edu.in
}

\begin{abstract}
Learning is based on collaborative learning theory. Collaborative learning theory has interaction, individual accountability, teamwork and personalized guidance. All these aspects can be performed in web 2.0 using social networking sites. So e-learning 2.0 on web 2.0 is not a new class of learning management system or a pedagogy but which promotes the user to collaborate and build the information and not just a mere spectator/consumer of information. In this paper the researchers have made an assessment of 23 e-learning systems, a survey on some of the popular tools/sites which will be useful and augment e-learning 2.0 and have discussed the features of an experimental web solution to have a look and feel of these tools.
\end{abstract}

Index Terms-E-Learning 2.0, Web 2.0, Learning Styles, Social Networking, Cloud Computing.

\section{INTRODUCTION}

An individual's way of processing information is said to be cognitive styles (CS) [1]. Humans have the cognitive ability to acquire and recognize information, map them to representations and then to knowledge and then use them. Some of the CS are Cognitive Trait Model and Inductive Reasoning Ability [2]. Learning Styles are the different ways in which learners perceive and process information [3]. Several learning styles have been in [4] towards designing e-learning materials based on multi-learner style [5]. Felder-Silverman's synthesis of these theories is designed to easily translate them into strategies for a higher education sector (Table I) [6]. Mostly the authors classify the learners into groups and propose corresponding inventories and methods. One such model is Sarasin [7]. According to him, the learners are classified into three groups based on their learning styles namely visual learners, auditory learners and kinesthetic learners. Visual learners gain knowledge through visual inputs, auditory learners learn through listening and kinesthetic learners learn through experiments and exploration [8].

This paper is structured as follows. Section II describes about e-Learning and lists some of the popular e-learn ing systems used around the world and provides a comparison table of these systems with FelderSilverman model. Section III identifies various ways of utilizing web 2.0 to augment e-Learning. Section IV and Section $\mathrm{V}$ lists the various tools in web 2.0 and compares its characteristics with the Felder-Silverman model and tabulates them. Section VI shows the proposed comprehensive e-LMS and discusses its features. Finally a brief conclusion is described in Section VII.

\section{E-LEARNING}

Teaching and learning process has undergone a vast change after the advent of Internet. As Internet and World Wide Web expanded rapidly e-Learning has become a major form of education where time and geographical constraints have been overcome. As pointed out in [9] e-Learning is a learning that involves acquisition, generation and transfer of knowledge using ICT. This enables universal learning, overcoming the barriers of time and distance. E-learning is based on learning objects [10] like audio, video, presentations, documents, etc., which are stored in a learning repository from where they are accessed and processed by the learner. The learning management systems like Black Board, Web CT, MOODLE etc., support various kinds of teaching learning activities but, they do not suit all kinds of learners who have different levels of intelligence and maintains "one size fits all” approach. Hence, the adaptive and intelligent web based educational systems [11], which cater to different types of styles of learning according to the requirements of the learner and their situations. Net Coach and SIETTE are comparable with modern day LMS. 
TABLE I: LEARNING STYLES

\begin{tabular}{|c|c|c|c|c|c|}
\hline Authors & \multicolumn{5}{|l|}{ Characteristic's } \\
\hline David k ol b’s & Accommodating & \multicolumn{2}{|l|}{ Diverg ing } & Converging & Assimilating \\
\hline Peter Honey \& Alan Mumford's & Activists & \multicolumn{2}{|l|}{ Reflectors } & Theorists & Prag matists \\
\hline Dunn and Dunn & Environmental & Emotional & Sociological & Physiological & Psychological \\
\hline Fel der-Silverman & $\begin{array}{l}\text { Active / } \\
\text { Reflective }\end{array}$ & \multicolumn{2}{|c|}{ Visual / Verbal } & $\begin{array}{l}\text { Sensing / } \\
\text { Intuitive }\end{array}$ & $\begin{array}{l}\text { Sequential / } \\
\text { Global }\end{array}$ \\
\hline Sarasin VAK & Visual & \multicolumn{3}{|l|}{ Auditory } & Kinesthetic \\
\hline
\end{tabular}

Most of the e-Learning systems, adaptive or non adaptive, do not relate or collaborate with external systems and mostly they are stand alone. A search was made for some of the popular e-learning management systems along with their URL (Table II). This motivated the researchers to make a comparison between the popular e-LMS's and the Felder-Silverman model. So, a comparative study was done on the systems mentioned in Table II for the various characteristics of the selected model to know how far they exhibit them. Table III provides these statistics. The serial number in Table III corresponds to the LMS listed in Table II.

\section{E-LEARNING IN WEB 2.0}

Web 2.0 is a collection of inter operable web applications that facilitate user interaction and collaboration with each other as creators of content rather than passive viewers as in web 1.0. Examples of Web 2.0 are social networking sites, blog, wikis, mashups, etc. [12]. Many of these tools can be used by the learning community to interact, share knowledge and augment problem based learning. It has ignited the culture of not only connection but also contribution. Web 1.0 was useful for connecting people to the contents thus useful for finding information and performing basic transactions. The meeting point of users and resources are called a course [13]. E-learning is primarily focused on groups. A group is a collection of individuals who are engaged in a joint work. One such place is a social networking site, which are very popular among people to stay connected. "Social” is often referred to as communication, construction and collaboration [13]. Currently 300 million people use social software [14] and it is still growing. In social networking, each individual has a page and profile that one develops and shares with others in the network. These social networking sites act as pedagogical agent for problem based learning since it is a combination of personalization and socialization. These social software provide platform to enable users to build applications for e-learning and enables them to learn from each others' work [14].

To broaden and generalize the data transfer between different social networking sites, Google brought out an open social development platform in 2007. This platform defines common API, which can be used to develop sites to provide services to multiple sites and easily exchange data between different social sites. MySpace, Friendster, LinkedIn uses open social API. Using REST API, personalized materials and queries can be provided to users. The dimensions of a high quality education experience are structure, content, service and delivery [15]. Structure is the foundation of quality content, service and delivery. Content should be comprehensive, authentic and researched. Services are resources, administration and technical support for the action done. Delivery is the usability, interactivity levels of the action performed [16]. All these desirable dimensions could be easily achieved through web 2.0. It is also very easy to monitor the students' learning process and levels through social media since they are open and transparent and allow the learners to construct their own learning. The critical challenge of web 2.0 is identifying the right co learners, content, service, and user interfaces to facilitate interaction, communication and collaboration. Scaling is another problem and expensive to implement. Here, Cloud computing comes to the rescue by providing storage and other services, freeing the users to focus on applications [17]. Applications like Google docs, presentation, etc., provide teachers and learners with free and low cost alternative. Browser based applications are easily accessible even on mobile platforms making learning possible where Internet is accessible.

\section{TOOLS OTHER THAN SOCIAL NETWORKING SITES}

Web 2.0 offers a wide variety of tools that can be used 
for e-learning. Social networking sites can be used for e-learning. According to Felder-Silverman Learning style model, the learn ing style can be sensory, intuitive, visual, verbal, inductive, deductive, active, reflective, sequential and global. There are a number of tools in web 2.0, which according to the researchers, could be mapped to Felder Silverman learning styles. Tools such as WebCasting and PodCasting can be used to broadcast audio/video/slide/recordings, which can complement classroom environment. Similarly, Wikis for group assignments; wikiversity.org to collaborate and create contents freely; SuTree.com and Slide.net offers a variety of resources to learners and teachers; eduSlide allows teachers to group their content and present them to the learners; Footnote allows the users to access documents and other resources and prepare online reports; VoiceThread.com allows tutors to create lessons and add audio narrative along with it;

TABLE II: LIST OF POPULAR E-LEARNING SYSTEMS

\begin{tabular}{|c|c|c|}
\hline $\begin{array}{l}\text { S. } \\
\text { No }\end{array}$ & Title & URL \\
\hline 1 & Apex Learning & www.ape xlearn ing.com/ \\
\hline 2 & ATutor & www.atutor.ca/credits.php \\
\hline 3 & Blackboard & www.blackboard.com/ \\
\hline 4 & Brainshark & www.brainshark.co m/ \\
\hline 5 & Chamilo & www.chamilo.org/ \\
\hline 6 & Claroline & www.c laro line.net/ \\
\hline 7 & CERTPOINT & www.certpointsystems.com/ \\
\hline 8 & Desire2Learn & www.desire 2lea rn.co m/ \\
\hline 9 & DoceboLMS & www.docebo.com/ \\
\hline 10 & Dokeos & www.dokeos.com/ \\
\hline 11 & LRN & www.dotlrn.org \\
\hline 12 & Latitude learning & www.latitudelearn ing.com/ \\
\hline 13 & EFront & www.e frontlearning.net/ \\
\hline 14 & HotChalk & www.hotchalk.com/ \\
\hline 15 & ILIAS & www.ilias.de/docu/ \\
\hline 16 & Interactyx & www.interacty x.co m/ \\
\hline 17 & Moodle & www.moodle.org/ \\
\hline 18 & Metacoon & www.metacoon.net/ \\
\hline 19 & $\begin{array}{l}\text { Meridian Knowledge } \\
\text { Solutions }\end{array}$ & www.meridianksi.com/ \\
\hline 20 & RCampus & www.rcampus.com/ \\
\hline 21 & Saba Software & www.saba.com/ \\
\hline 22 & Sakai & www.saka iproject.org/ \\
\hline 23 & Studywiz & www.studywiz.co m/ \\
\hline
\end{tabular}

TABLE III: FELDER-SIL VERM AN MODEL vs ELEARNING SYSTEMS

\begin{tabular}{|c|c|c|c|c|c|c|c|c|}
\hline$\sum_{=}^{\infty}$ & 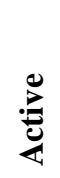 & 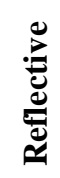 & $\stackrel{\pi}{3}$ & गे & ڤొ & 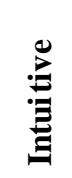 & 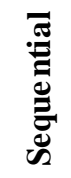 & 중 \\
\hline 1 & $\mathrm{~N}$ & $\mathrm{Y}$ & $\mathrm{P}$ & $\mathrm{N}$ & $\mathrm{Y}$ & $\mathrm{P}$ & $\mathrm{Y}$ & $\mathrm{Y}$ \\
\hline 2 & $\mathrm{Y}$ & $\mathrm{Y}$ & $\mathrm{P}$ & $\mathrm{Y}$ & $\mathrm{Y}$ & $\mathrm{P}$ & $\mathrm{Y}$ & $\mathrm{Y}$ \\
\hline 3 & $\mathrm{P}$ & $\mathrm{Y}$ & $\mathrm{P}$ & $\mathrm{P}$ & $\mathrm{Y}$ & $\mathrm{P}$ & $\mathrm{Y}$ & $\mathrm{Y}$ \\
\hline 4 & $\mathrm{~N}$ & $\mathrm{Y}$ & $\mathrm{Y}$ & $\mathrm{N}$ & $\mathrm{Y}$ & $\mathrm{P}$ & $\mathrm{P}$ & $\mathrm{Y}$ \\
\hline 5 & $\mathrm{Y}$ & $\mathrm{Y}$ & $\mathrm{P}$ & $\mathrm{Y}$ & $\mathrm{Y}$ & $\mathrm{Y}$ & $\mathrm{Y}$ & $\mathrm{Y}$ \\
\hline 6 & $\mathrm{Y}$ & $\mathrm{P}$ & $\mathrm{P}$ & $\mathrm{P}$ & $\mathrm{Y}$ & $\mathrm{Y}$ & $\mathrm{Y}$ & $\mathrm{Y}$ \\
\hline 7 & $\mathrm{P}$ & $\mathrm{Y}$ & $\mathrm{Y}$ & $\mathrm{P}$ & $\mathrm{Y}$ & $\mathrm{Y}$ & $\mathrm{Y}$ & $\mathrm{Y}$ \\
\hline 8 & $\mathrm{~N}$ & $\mathrm{Y}$ & $\mathrm{Y}$ & $\mathrm{N}$ & $\mathrm{Y}$ & $\mathrm{Y}$ & $\mathrm{P}$ & $\mathrm{Y}$ \\
\hline 9 & $\mathrm{Y}$ & $\mathrm{Y}$ & $\mathrm{Y}$ & $\mathrm{P}$ & $\mathrm{Y}$ & $\mathrm{Y}$ & $\mathrm{P}$ & $\mathrm{Y}$ \\
\hline 10 & $\mathrm{Y}$ & $\mathrm{Y}$ & $\mathrm{P}$ & $\mathrm{Y}$ & $\mathrm{Y}$ & $\mathrm{Y}$ & $\mathrm{Y}$ & $\mathrm{Y}$ \\
\hline 11 & $\mathrm{Y}$ & $\mathrm{Y}$ & $\mathrm{P}$ & $\mathrm{P}$ & $\mathrm{Y}$ & $\mathrm{Y}$ & $\mathrm{Y}$ & $\mathrm{Y}$ \\
\hline 12 & $\mathrm{Y}$ & $\mathrm{Y}$ & $\mathrm{Y}$ & $\mathrm{Y}$ & $\mathrm{Y}$ & $\mathrm{Y}$ & $\mathrm{Y}$ & $\mathrm{Y}$ \\
\hline 13 & $\mathrm{Y}$ & $\mathrm{Y}$ & $\mathrm{P}$ & $\mathrm{Y}$ & $\mathrm{Y}$ & $\mathrm{Y}$ & $\mathrm{Y}$ & $\mathrm{Y}$ \\
\hline 14 & $\mathrm{Y}$ & $\mathrm{Y}$ & $\mathrm{Y}$ & $\mathrm{Y}$ & $\mathrm{Y}$ & $\mathrm{Y}$ & $\mathrm{Y}$ & $\mathrm{Y}$ \\
\hline 15 & $\mathrm{Y}$ & $\mathrm{Y}$ & $\mathrm{Y}$ & $\mathrm{Y}$ & $\mathrm{Y}$ & $\mathrm{Y}$ & $\mathrm{Y}$ & $\mathrm{Y}$ \\
\hline 16 & $\mathrm{Y}$ & $\mathrm{Y}$ & $\mathrm{P}$ & $\mathrm{P}$ & $\mathrm{Y}$ & $\mathrm{Y}$ & $\mathrm{Y}$ & $\mathrm{Y}$ \\
\hline 17 & $\mathrm{Y}$ & $\mathrm{Y}$ & $\mathrm{Y}$ & $\mathrm{Y}$ & $\mathrm{Y}$ & $\mathrm{Y}$ & $\mathrm{Y}$ & $\mathrm{Y}$ \\
\hline 18 & $\mathrm{Y}$ & $\mathrm{Y}$ & $\mathrm{P}$ & $\mathrm{P}$ & $\mathrm{Y}$ & $\mathrm{Y}$ & $\mathrm{Y}$ & $\mathrm{Y}$ \\
\hline 19 & $\mathrm{~N}$ & $\mathrm{Y}$ & $\mathrm{P}$ & $\mathrm{P}$ & $\mathrm{Y}$ & $\mathrm{Y}$ & $\mathrm{Y}$ & $\mathrm{Y}$ \\
\hline 20 & $\mathrm{Y}$ & $\mathrm{Y}$ & $\mathrm{Y}$ & $\mathrm{P}$ & $\mathrm{Y}$ & $\mathrm{Y}$ & $\mathrm{Y}$ & $\mathrm{Y}$ \\
\hline 21 & $\mathrm{~N}$ & $\mathrm{Y}$ & $\mathrm{P}$ & $\mathrm{P}$ & $\mathrm{Y}$ & $\mathrm{Y}$ & $\mathrm{Y}$ & $\mathrm{Y}$ \\
\hline 22 & $\mathrm{Y}$ & $\mathrm{Y}$ & $\mathrm{Y}$ & $\mathrm{Y}$ & $\mathrm{Y}$ & $\mathrm{Y}$ & $\mathrm{Y}$ & $\mathrm{Y}$ \\
\hline 23 & $\mathrm{~N}$ & $\mathrm{Y}$ & $\mathrm{P}$ & $\mathrm{P}$ & $\mathrm{Y}$ & $\mathrm{Y}$ & $\mathrm{Y}$ & $\mathrm{Y}$ \\
\hline
\end{tabular}

Y - Yes, Fully Available, P - Partially Available, $N$ No, Not Available

Visual Thesarus.com provides all the synonyms in graphical format; and wePapers.com allows sharing of academic/research papers, tutorials, lectures and test. LMS are learning management systems, which provide the learner with the learning contents. Personal Learning Environments (PLE) are tools, used by the learners to gather information, reflect and work on them. Since these tools are not exclusively meant for LMS, they could lead one to error. So, it becomes necessary to provide a system with the comb ination of LMS and PLE under controlled environment and this process can be done using web 2.0 tools. For example, SHARek - uses social bookmarking and consists of PLE within LMS. 


\section{TOOLS IN WEB 2.0}

A survey was made to look for e-Learning components such as repository to hold different items like learning objects, student profile, etc., multimedia, simulations, gaming, quizzing, searching, socializing, assessment, etc., as mentioned in Felder-Silverman style of learning. Some of the popular sites/tools which can be used for e-Learning are tabulated in Table IV.

TABLE IV: SITES SUR VEYED

\begin{tabular}{|c|c|}
\hline S.No & Websites / Tools \\
\hline 1 & https://voicethread.com/ \\
\hline 2 & www.facebook.com \\
\hline 3 & www.myspace.com \\
\hline 4 & www.t witter.com \\
\hline 5 & http://www. wayfaring.com/ \\
\hline 6 & http://www.wepapers.com/ \\
\hline 7 & http://www.wordpress.com/ \\
\hline 8 & http://www.bebo.com/ \\
\hline 9 & http://www.ning.co m/ \\
\hline 10 & http://www.friendster.com/ \\
\hline 11 & http://www.linkedin.co m/ \\
\hline 12 & http://www.stumbleupon.com/ \\
\hline 13 & www.coolprev iews.com \\
\hline 14 & www.wikiversity.org \\
\hline 15 & www.v isualthes aurus.com \\
\hline 16 & http://translate.google.co.in \\
\hline 17 & http://maps.google.co.in/ \\
\hline 18 & www.classmarker.com \\
\hline 19 & www.quizga laxy.com \\
\hline 20 & www.tcyonline.com \\
\hline 21 & www.surveymonkey.com \\
\hline 22 & www.zoo merang.com \\
\hline 23 & http://www.s mg2000.org/ \\
\hline 24 & www.marketwatch.com/ \\
\hline 25 & www.cut-the-knot.org/ \\
\hline 26 & http://lstat.kuleuven.be/java/ \\
\hline
\end{tabular}

\begin{tabular}{|c|c|}
\hline 27 & $\begin{array}{l}\text { http://www.this waytocpa.com/career- } \\
\text { tools/interview-simulation/ }\end{array}$ \\
\hline 28 & http://en.wikipedia.org/wiki/Webcast \\
\hline 29 & http://www.librivo x.org/ \\
\hline 30 & www.slideshare.com \\
\hline 31 & www.scribd.com \\
\hline 32 & www.docs.google.com \\
\hline 33 & www.youtube.com \\
\hline 34 & $\begin{array}{l}\text { http://www.google.co.in/blogsearch?hl=en\&ta } \\
\text { b=wb }\end{array}$ \\
\hline 35 & http://pipes.yahoo.com/pipes/ \\
\hline 36 & http://www.programmable web.com/ \\
\hline 37 & http://www.google.co m/talk/ \\
\hline 38 & https://www.google.co m/calendar/ \\
\hline 39 & http://trans late.google.com/ \\
\hline 40 & https://sites.google.com/ \\
\hline 41 & http://www.google.co.in/ig \\
\hline 42 & www.rapidshare.com \\
\hline 43 & www.mediafire.com \\
\hline 44 & www.hotfile.com \\
\hline 45 & www.4shared.com \\
\hline 46 & http://depositfiles.com/ \\
\hline 47 & http://zshare.net/ \\
\hline 48 & http://uploading.com/ \\
\hline 49 & $\begin{array}{l}\text { http://www.csse.monash.edu.au/ dwa/Animati } \\
\text { ons/index.html }\end{array}$ \\
\hline 50 & $\begin{array}{l}\text { http://www.cosc.canterbury.ac.nz/mu kundan/d } \\
\text { sal/appldsal.html }\end{array}$ \\
\hline 51 & $\begin{array}{l}\text { http://www.cs.jhu.edu/ goodrich/dsa/trees/ind } \\
\text { ex.html }\end{array}$ \\
\hline 52 & $\begin{array}{l}\text { http://downloads.ziddu.com/downloadfile/123 } \\
\text { 14754/Data_Structures_Through_C_- } \\
\text { Yashavant_Kanetkar.rar.html }\end{array}$ \\
\hline 53 & $\begin{array}{l}\text { http://www.d zone.com/lin ks/animated_tutorial } \\
\text { s_for_data_structure_algorithms.html }\end{array}$ \\
\hline 54 & http://www.edsim51.com/ \\
\hline 55 & $\begin{array}{l}\text { http://www.ho mes oft.gen.tr/products/Sim8085 } \\
\text {.html }\end{array}$ \\
\hline 56 & http://sourceforge.net/projects/picmicrosim/ \\
\hline
\end{tabular}




\begin{tabular}{|c|c|}
\hline 57 & $\begin{array}{l}\text { http://www.angelfire.co m/electronic 2/8085sim } \\
\text { ulator/ }\end{array}$ \\
\hline 58 & http://www.samphire.demon.co.uk/ \\
\hline 59 & http://www.ontko.com/moss/ \\
\hline 60 & http://vip.cs.utsa.edu/simulators/ \\
\hline 61 & $\begin{array}{l}\text { http://www.cs.kent.ac.uk/people/staff/frmb/mo } \\
\text { ss/ }\end{array}$ \\
\hline 62 & $\begin{array}{l}\text { http://www.cs.unm.edu/ crowley/osbook/sos.h } \\
\text { tml }\end{array}$ \\
\hline 63 & $\begin{array}{l}\text { http://www. freevbcode.com/ShowCode.asp?I } \\
\mathrm{D}=4079\end{array}$ \\
\hline 64 & $\begin{array}{l}\text { http://samate.nist.gov/index.php/Tool_Survey. } \\
\text { html }\end{array}$ \\
\hline 65 & http://pmd.sourceforge.net/ \\
\hline 66 & $\begin{array}{l}\text { http://www.stachliu.com/resources/tools/googl } \\
\text { e-hacking-diggity-project/attack-tools/ }\end{array}$ \\
\hline 67 & http://findbugs sourceforge.net/ \\
\hline 68 & $\begin{array}{l}\text { https://www. fortify.co m/ssa-elements/threat- } \\
\text { intelligence/rats.html }\end{array}$ \\
\hline 69 & $\begin{array}{l}\text { http://www.modelsphere.org/open_modelsphe } \\
\text { re.html }\end{array}$ \\
\hline 70 & http://www.sqlpower.ca/page/architect \\
\hline 71 & http://www.dbvis.com/ \\
\hline 72 & http://www.sqlmanager.net/tools/free \\
\hline 73 & http://www.elphsoft.com/ \\
\hline 74 & http://www.isi.edu/nsnam/ns/ \\
\hline 75 & $\begin{array}{l}\text { http://www.cc.gatech.edu/computing/compass/ } \\
\text { pdns/index.html }\end{array}$ \\
\hline 76 & http://www.ss fnet.org/homePage.html \\
\hline 77 & $\begin{array}{l}\text { http://www.cisco.com/web/learn ing/netacad/c } \\
\text { ourse_catalog/PacketTracer.html }\end{array}$ \\
\hline 78 & $\begin{array}{l}\text { http://www.filebuzz.co m/findsoftware/Free_N } \\
\text { etwork_Simulation_Exe/1.html }\end{array}$ \\
\hline 79 & www.questionwriter.org \\
\hline 80 & www.co mpilr.com \\
\hline 81 & www.spicynodes.com \\
\hline
\end{tabular}

The websites surveyed exhibit the following features, which corresponds to Felder-Silverman learning styles. These are collaboration; sharing materials; creating groups / communities; uploading, managing and downloading materials / files; creating web pages / blogs; chatting/broadcast - audio, video, text, search and discover; perform analytics; play games; perform testing and evaluation of the learner; track his learning pattern; allow the user to experiment / simulate different scenarios; conduct a survey and gain insight; translate to different languages; make use of open and free educational resources; and provide free storage and more.

A comparison of matrix between the FelderSilverman learning style and the sites survey is illustrated in Table V. The extreme left vertical column identifies the sites by its serial number as mentioned in Table IV and the top most horizontal row represents the eight learning styles from 'a to i' as mentioned in Table I. A mark ' $Y$ ' is made, if the feature is available in the site, ' $\mathrm{N}$ ' if not available and ' $\mathrm{P}$ ' if partially available.

TABLE V: COMPARISON BETWEEN FELDERSILVERM AN MODEL AND TOOLS IN WEB 2.0

\begin{tabular}{|c|c|c|c|c|c|c|c|c|}
\hline $\begin{array}{l}\text { Tools / Felder - } \\
\text { Silverman Model }\end{array}$ & 趂。 & $\bar{\Xi}$ & & & & $\because$ & $\stackrel{\Xi}{\varrho}$ & \\
\hline $\begin{array}{l}\text { http://voicethread.c } \\
\text { om/ }\end{array}$ & $Y$ & $Y$ & $Y$ & $\mathrm{~N}$ & $\mathrm{P}$ & $\mathrm{Y}$ & $\mathrm{Y}$ & $\mathrm{P}$ \\
\hline $\begin{array}{l}\text { https://www.facebo } \\
\text { ok.com/ }\end{array}$ & $\mathrm{Y}$ & $Y$ & $Y$ & $Y$ & $\mathrm{Y}$ & $\mathrm{Y}$ & $\mathrm{P}$ & $\mathrm{Y}$ \\
\hline $\begin{array}{l}\text { https://new.myspac } \\
\text { e.com/ }\end{array}$ & $P$ & $P$ & $\mathrm{Y}$ & Y & $P$ & $\mathrm{P}$ & $\mathrm{N}$ & $\mathrm{Y}$ \\
\hline $\begin{array}{l}\text { https://www.t witter } \\
\text {.com/ }\end{array}$ & $Y$ & $Y$ & $\mathrm{P}$ & $P$ & $\mathrm{Y}$ & $\mathrm{Y}$ & $\mathrm{P}$ & $\mathrm{Y}$ \\
\hline $\begin{array}{l}\text { http://www. wayfari } \\
\text { ng.com/ }\end{array}$ & $\mathrm{Y}$ & $Y$ & $\mathrm{P}$ & $\mathrm{N}$ & $\mathrm{P}$ & $\mathrm{P}$ & $\mathrm{Y}$ & $\mathrm{N}$ \\
\hline $\begin{array}{l}\text { http://www.wepape } \\
\text { rs.com/ }\end{array}$ & $\mathrm{Y}$ & $Y$ & $\mathrm{~N}$ & $\mathrm{~N}$ & $\mathrm{~N}$ & $\mathrm{~N}$ & $\mathrm{P}$ & $\mathrm{Y}$ \\
\hline $\begin{array}{l}\text { http://wordpress.co } \\
\mathrm{m} /\end{array}$ & $Y$ & $\mathrm{Y}$ & $Y$ & $Y$ & $\mathrm{Y}$ & $\mathrm{P}$ & $\mathrm{Y}$ & $\mathrm{P}$ \\
\hline $\begin{array}{l}\text { http://www.bebo.c } \\
\text { om/ }\end{array}$ & $\mathrm{Y}$ & $\mathrm{Y}$ & $Y$ & $Y$ & $\mathrm{P}$ & $\mathrm{N}$ & $\mathrm{P}$ & $Y$ \\
\hline $\begin{array}{l}\text { http://www.ning.co } \\
\text { m/ }\end{array}$ & $Y$ & $\mathrm{Y}$ & $Y$ & $Y$ & $\mathrm{Y}$ & $\mathrm{P}$ & $\mathrm{P}$ & $Y$ \\
\hline $\begin{array}{l}\text { http://www.friendst } \\
\text { er.com/ }\end{array}$ & $Y$ & $\mathrm{Y}$ & $\mathrm{P}$ & $\mathrm{N}$ & $\mathrm{Y}$ & $\mathrm{P}$ & $\mathrm{N}$ & $\mathrm{Y}$ \\
\hline $\begin{array}{l}\text { http://in.linked in.c } \\
\text { om/ }\end{array}$ & $Y$ & $\mathrm{Y}$ & $\mathrm{P}$ & $\mathrm{N}$ & $\mathrm{Y}$ & $\mathrm{N}$ & $\mathrm{N}$ & $\mathrm{Y}$ \\
\hline $\begin{array}{l}\text { https://www.stumb } \\
\text { leupon.com/ }\end{array}$ & $Y$ & $Y$ & $Y$ & $Y$ & $\mathrm{~N}$ & $\mathrm{~N}$ & $\mathrm{P}$ & $\mathrm{Y}$ \\
\hline $\begin{array}{l}\text { http://www.coolpre } \\
\text { views.com/ }\end{array}$ & $\mathrm{P}$ & $Y$ & $\mathrm{~N}$ & $\mathrm{P}$ & $\mathrm{N}$ & $\mathrm{N}$ & $\mathrm{Y}$ & $\mathrm{P}$ \\
\hline $\begin{array}{l}\text { http://www. wikiver } \\
\text { sity.org/ }\end{array}$ & $\mathrm{P}$ & $Y$ & $Y$ & $Y$ & $\mathrm{P}$ & $\mathrm{P}$ & $\mathrm{Y}$ & $\mathrm{P}$ \\
\hline $\begin{array}{l}\text { http://www.visualt } \\
\text { hesaurus.com/ }\end{array}$ & $Y$ & $Y$ & $Y$ & $Y$ & $\mathrm{~N}$ & $\mathrm{P}$ & $\mathrm{Y}$ & $\mathrm{P}$ \\
\hline $\begin{array}{l}\text { http://trans late.goo } \\
\text { gle.co.in/ }\end{array}$ & $\mathrm{Y}$ & $Y$ & $\mathrm{~N}$ & $Y$ & $Y$ & $\mathrm{P}$ & $\mathrm{P}$ & $Y$ \\
\hline $\begin{array}{l}\text { http://maps.google. } \\
\text { co.in/ }\end{array}$ & $\mathrm{N}$ & $\mathrm{Y}$ & $Y$ & $\mathrm{~N}$ & $Y$ & $\mathrm{~N}$ & $\mathrm{~N}$ & $Y$ \\
\hline $\begin{array}{l}\text { http://www.classm } \\
\text { arker.com/ }\end{array}$ & $Y$ & $Y$ & $\mathrm{P}$ & $Y$ & $Y$ & $\mathrm{Y}$ & $\mathrm{Y}$ & $\mathrm{P}$ \\
\hline $\begin{array}{l}\text { http://www.quizgal } \\
\text { axy.com/ }\end{array}$ & $\mathrm{N}$ & $\mathrm{Y}$ & $\mathrm{P}$ & $\mathrm{P}$ & $\mathrm{P}$ & $\mathrm{P}$ & $\mathrm{Y}$ & $\mathrm{P}$ \\
\hline
\end{tabular}




\begin{tabular}{|c|c|c|c|c|c|c|c|c|}
\hline $\begin{array}{l}\text { http://www.tcyonli } \\
\text { ne.com/ }\end{array}$ & $\mathrm{N}$ & $\mathrm{Y}$ & $\mathrm{P}$ & $\mathrm{P}$ & $\mathrm{Y}$ & $\mathrm{P}$ & $\mathrm{P}$ & $\mathrm{Y}$ \\
\hline $\begin{array}{l}\text { http://www.survey } \\
\text { monkey.com/ }\end{array}$ & $\mathrm{Y}$ & $\mathrm{Y}$ & $\mathrm{Y}$ & $\mathrm{N}$ & $\mathrm{P}$ & $\mathrm{P}$ & $\mathrm{Y}$ & $\mathrm{P}$ \\
\hline $\begin{array}{l}\text { http://www.zoo mer } \\
\text { ang.com/ }\end{array}$ & $\mathrm{Y}$ & $\mathrm{Y}$ & $\mathrm{Y}$ & $\mathrm{N}$ & $\mathrm{P}$ & $\mathrm{P}$ & $\mathrm{Y}$ & $\mathrm{P}$ \\
\hline $\begin{array}{l}\text { http://www.s mg20 } \\
\text { 00.org }\end{array}$ & $\mathrm{Y}$ & $\mathrm{Y}$ & $\mathrm{Y}$ & $\mathrm{N}$ & $\mathrm{N}$ & $\mathrm{Y}$ & $\mathrm{Y}$ & $\mathrm{P}$ \\
\hline $\begin{array}{l}\text { http://www.market } \\
\text { watch.com/ }\end{array}$ & $\mathrm{P}$ & $\mathrm{Y}$ & $\mathrm{Y}$ & $\mathrm{P}$ & $\mathrm{Y}$ & $\mathrm{P}$ & $\mathrm{Y}$ & $\mathrm{P}$ \\
\hline $\begin{array}{l}\text { http://www.cut- } \\
\text { the-knot.org/ }\end{array}$ & $\mathrm{N}$ & $\mathrm{Y}$ & $\mathrm{Y}$ & $\mathrm{P}$ & $\mathrm{P}$ & $\mathrm{P}$ & $\mathrm{Y}$ & $\mathrm{P}$ \\
\hline $\begin{array}{l}\text { http://lstat.kuleuve } \\
\text { n.be/java/ }\end{array}$ & $\mathrm{N}$ & $\mathrm{Y}$ & $\mathrm{Y}$ & $\mathrm{N}$ & $\mathrm{Y}$ & $\mathrm{P}$ & $\mathrm{Y}$ & $\mathrm{P}$ \\
\hline $\begin{array}{l}\text { https://www.this wa } \\
\text { ytocpa.com/career- } \\
\text { tools/interview- } \\
\text { simu lation/ } \\
\end{array}$ & $\mathrm{Y}$ & $\mathrm{Y}$ & $\mathrm{Y}$ & $\mathrm{Y}$ & $\mathrm{N}$ & $\mathrm{P}$ & $\mathrm{N}$ & $\mathrm{Y}$ \\
\hline $\begin{array}{l}\text { http://en.wikipedia. } \\
\text { org/wiki/Webcast }\end{array}$ & $\mathrm{N}$ & $\mathrm{Y}$ & $\mathrm{Y}$ & $\mathrm{Y}$ & $\mathrm{P}$ & $\mathrm{P}$ & $\mathrm{Y}$ & $\mathrm{P}$ \\
\hline http://librivo x.org/ & $\mathrm{N}$ & $\mathrm{Y}$ & $\mathrm{N}$ & $\mathrm{Y}$ & $\mathrm{N}$ & $\mathrm{Y}$ & $\mathrm{Y}$ & $\mathrm{N}$ \\
\hline $\begin{array}{l}\text { http://www.slidesh } \\
\text { are.net/ }\end{array}$ & $\mathrm{Y}$ & $\mathrm{Y}$ & $\mathrm{Y}$ & $\mathrm{N}$ & $\mathrm{N}$ & $\mathrm{N}$ & $\mathrm{Y}$ & $\mathrm{P}$ \\
\hline $\begin{array}{l}\text { http://www.s cribd. } \\
\text { com/ }\end{array}$ & $\mathrm{Y}$ & $\mathrm{Y}$ & $\mathrm{Y}$ & $\mathrm{N}$ & $\mathrm{N}$ & $\mathrm{P}$ & $\mathrm{P}$ & $\mathrm{Y}$ \\
\hline $\begin{array}{l}\text { http://docs.google. } \\
\text { com/ }\end{array}$ & $\mathrm{Y}$ & $\mathrm{Y}$ & $\mathrm{Y}$ & $\mathrm{N}$ & $\mathrm{Y}$ & $\mathrm{Y}$ & $\mathrm{Y}$ & $\mathrm{P}$ \\
\hline $\begin{array}{l}\text { http://www.youtub } \\
\text { e.com/ }\end{array}$ & $\mathrm{Y}$ & $\mathrm{Y}$ & $\mathrm{Y}$ & $\mathrm{Y}$ & $\mathrm{N}$ & $\mathrm{N}$ & $\mathrm{P}$ & $\mathrm{Y}$ \\
\hline $\begin{array}{l}\text { http://www.google. } \\
\text { com/blogsearch?hl } \\
\text { =en\&tab=wb }\end{array}$ & $\mathrm{Y}$ & $Y$ & $\mathrm{P}$ & $\mathrm{P}$ & $Y$ & $\mathrm{P}$ & $Y$ & $\mathrm{~N}$ \\
\hline $\begin{array}{l}\text { http://pipes.yahoo. } \\
\text { com/pipes/ }\end{array}$ & $\mathrm{P}$ & $\mathrm{Y}$ & $\mathrm{Y}$ & $\mathrm{N}$ & $\mathrm{Y}$ & $\mathrm{Y}$ & $\mathrm{Y}$ & $\mathrm{P}$ \\
\hline $\begin{array}{l}\text { http://www.progra } \\
\text { mmable web.com// }\end{array}$ & $\mathrm{Y}$ & $\mathrm{Y}$ & $\mathrm{Y}$ & $\mathrm{N}$ & $\mathrm{P}$ & $\mathrm{Y}$ & $\mathrm{Y}$ & $\mathrm{P}$ \\
\hline $\begin{array}{l}\text { http://www.google. } \\
\text { co.in/talk/ }\end{array}$ & $\mathrm{Y}$ & $\mathrm{Y}$ & $\mathrm{Y}$ & $\mathrm{Y}$ & $\mathrm{P}$ & $\mathrm{P}$ & $\mathrm{Y}$ & $\mathrm{P}$ \\
\hline $\begin{array}{l}\text { https://www.googl } \\
\text { e.com/calendar }\end{array}$ & $\mathrm{Y}$ & $\mathrm{Y}$ & $\mathrm{Y}$ & $\mathrm{N}$ & $\mathrm{Y}$ & $\mathrm{Y}$ & $\mathrm{Y}$ & $\mathrm{P}$ \\
\hline $\begin{array}{l}\text { https://sites.google. } \\
\text { com/ }\end{array}$ & $\mathrm{Y}$ & $\mathrm{Y}$ & $\mathrm{Y}$ & $\mathrm{Y}$ & $Y$ & $\mathrm{Y}$ & $\mathrm{Y}$ & $\mathrm{P}$ \\
\hline $\begin{array}{l}\text { http://www.google. } \\
\text { com/ig }\end{array}$ & $\mathrm{Y}$ & $\mathrm{Y}$ & $\mathrm{Y}$ & $\mathrm{Y}$ & $\mathrm{N}$ & $\mathrm{Y}$ & $\mathrm{Y}$ & $\mathrm{P}$ \\
\hline $\begin{array}{l}\text { https://rapidshare.c } \\
\text { om/ }\end{array}$ & $\mathrm{Y}$ & $\mathrm{Y}$ & $\mathrm{Y}$ & $\mathrm{Y}$ & $\mathrm{Y}$ & $\mathrm{Y}$ & $\mathrm{Y}$ & $\mathrm{P}$ \\
\hline $\begin{array}{l}\text { http://www.mediafi } \\
\text { re.com/ }\end{array}$ & $\mathrm{Y}$ & $\mathrm{Y}$ & $\mathrm{Y}$ & $\mathrm{Y}$ & $\mathrm{Y}$ & $\mathrm{Y}$ & $\mathrm{Y}$ & $\mathrm{P}$ \\
\hline $\begin{array}{l}\text { http://www.hotfile. } \\
\text { com/ }\end{array}$ & $\mathrm{Y}$ & $\mathrm{Y}$ & $\mathrm{Y}$ & $\mathrm{Y}$ & $\mathrm{Y}$ & $\mathrm{Y}$ & $\mathrm{Y}$ & $\mathrm{P}$ \\
\hline $\begin{array}{l}\text { https://www.4share } \\
\text { d.com/ }\end{array}$ & $\mathrm{Y}$ & $\mathrm{Y}$ & $\mathrm{Y}$ & $\mathrm{Y}$ & $\mathrm{Y}$ & $\mathrm{Y}$ & $\mathrm{Y}$ & $\mathrm{P}$ \\
\hline $\begin{array}{l}\text { http://depositfiles.c } \\
\text { om/ }\end{array}$ & $\mathrm{Y}$ & $\mathrm{Y}$ & $\mathrm{Y}$ & $\mathrm{Y}$ & $\mathrm{Y}$ & $\mathrm{Y}$ & $\mathrm{Y}$ & $\mathrm{P}$ \\
\hline http://zshare.com/ & $\mathrm{Y}$ & $\mathrm{Y}$ & $\mathrm{Y}$ & $\mathrm{Y}$ & $\mathrm{Y}$ & $\mathrm{Y}$ & $\mathrm{Y}$ & $\mathrm{P}$ \\
\hline $\begin{array}{l}\text { http://uploading.co } \\
\mathrm{m} /\end{array}$ & $\mathrm{Y}$ & $\mathrm{Y}$ & $\mathrm{Y}$ & $\mathrm{Y}$ & $\mathrm{Y}$ & $\mathrm{Y}$ & $\mathrm{Y}$ & $\mathrm{P}$ \\
\hline $\begin{array}{l}\text { http://www.csse.m } \\
\text { onash.edu.au/ dwa }\end{array}$ & $\mathrm{N}$ & $\mathrm{Y}$ & $\mathrm{P}$ & $\mathrm{N}$ & $\mathrm{Y}$ & $\mathrm{Y}$ & $\mathrm{P}$ & $\mathrm{Y}$ \\
\hline
\end{tabular}

\begin{tabular}{|c|c|c|c|c|c|c|c|c|}
\hline $\begin{array}{l}\text { /Animations/index. } \\
\text { html }\end{array}$ & & & & & & & & \\
\hline $\begin{array}{l}\text { http://www.cosc.ca } \\
\text { nterbury.ac.nz/muk } \\
\text { undan/dsal/appldsa } \\
\text { l.html }\end{array}$ & $\mathrm{N}$ & $\mathrm{Y}$ & $\mathrm{P}$ & $\mathrm{N}$ & $\mathrm{P}$ & $Y$ & $\mathrm{P}$ & $\mathrm{Y}$ \\
\hline $\begin{array}{l}\text { http://www.cs.jhu.e } \\
\text { du/ goodrich/dsa/t } \\
\text { rees/index.html }\end{array}$ & $\mathrm{N}$ & $\mathrm{Y}$ & $\mathrm{P}$ & $\mathrm{N}$ & $\mathrm{N}$ & $\mathrm{Y}$ & $\mathrm{P}$ & $\mathrm{Y}$ \\
\hline $\begin{array}{l}\text { http://www.ziddu.c } \\
\text { om/download/1231 } \\
\text { 4754/Data_Structu } \\
\text { res_Through_C_- } \\
\text { Yashavant_Kanetk } \\
\text { ar.rar.html } \\
\end{array}$ & $\mathrm{N}$ & $\mathrm{Y}$ & $\mathrm{N}$ & $\mathrm{N}$ & $\mathrm{N}$ & $Y$ & $\mathrm{Y}$ & $\mathrm{N}$ \\
\hline $\begin{array}{l}\text { http://www.dzone. } \\
\text { com/links/animate } \\
\text { d_tutorials_for_dat } \\
\text { a_structure_algorit } \\
\text { hms.html }\end{array}$ & $\mathrm{P}$ & $\mathrm{Y}$ & $\mathrm{P}$ & $\mathrm{N}$ & $\mathrm{P}$ & $Y$ & $\mathrm{Y}$ & $\mathrm{N}$ \\
\hline $\begin{array}{l}\text { http://www.edsim5 } \\
\text { 1.com/ }\end{array}$ & $\mathrm{N}$ & $\mathrm{Y}$ & $\mathrm{P}$ & $\mathrm{N}$ & $\mathrm{Y}$ & $\mathrm{Y}$ & $\mathrm{Y}$ & $\mathrm{P}$ \\
\hline $\begin{array}{l}\text { http://www.homes } \\
\text { oft.gen.tr/products/ } \\
\text { Sim8085.html }\end{array}$ & $\mathrm{N}$ & $\mathrm{Y}$ & $\mathrm{P}$ & $\mathrm{N}$ & $\mathrm{N}$ & $\mathrm{P}$ & $\mathrm{Y}$ & $\mathrm{P}$ \\
\hline $\begin{array}{l}\text { http://sourceforge.n } \\
\text { et/projects/picmicr } \\
\text { osim/ }\end{array}$ & $\mathrm{N}$ & $\mathrm{Y}$ & $\mathrm{N}$ & $\mathrm{N}$ & $\mathrm{N}$ & $\mathrm{P}$ & $\mathrm{Y}$ & $\mathrm{P}$ \\
\hline $\begin{array}{l}\text { http://www.angelfi } \\
\text { re.co m/electronic } 2 / \\
\text { 8085simulator/ }\end{array}$ & $\mathrm{N}$ & $\mathrm{Y}$ & $\mathrm{Y}$ & $\mathrm{P}$ & $\mathrm{N}$ & $\mathrm{P}$ & $\mathrm{Y}$ & $\mathrm{P}$ \\
\hline $\begin{array}{l}\text { http://www.samphi } \\
\text { re.demon.co.uk/ }\end{array}$ & $\mathrm{N}$ & $\mathrm{Y}$ & $\mathrm{N}$ & $\mathrm{N}$ & $\mathrm{N}$ & $\mathrm{P}$ & $\mathrm{Y}$ & $\mathrm{P}$ \\
\hline $\begin{array}{l}\text { http://www.ontko.c } \\
\text { om/moss/ }\end{array}$ & $\mathrm{N}$ & $\mathrm{Y}$ & $\mathrm{N}$ & $\mathrm{N}$ & $\mathrm{N}$ & $Y$ & $\mathrm{Y}$ & $\mathrm{N}$ \\
\hline $\begin{array}{l}\text { http://vip.cs.utsa.ed } \\
\text { u/simulators/ }\end{array}$ & $\mathrm{N}$ & $\mathrm{Y}$ & $\mathrm{N}$ & $\mathrm{N}$ & $\mathrm{N}$ & $\mathrm{Y}$ & $\mathrm{Y}$ & $\mathrm{N}$ \\
\hline $\begin{array}{l}\text { http://www.cs.kent. } \\
\text { ac.uk/people/staff/f } \\
\text { rmb/moss/ }\end{array}$ & $\mathrm{N}$ & $\mathrm{Y}$ & $\mathrm{N}$ & $\mathrm{N}$ & $\mathrm{P}$ & $\mathrm{Y}$ & $\mathrm{Y}$ & $\mathrm{P}$ \\
\hline $\begin{array}{l}\text { http://www.cs.unm } \\
\text {.edu/ crowley/osb } \\
\text { ook/sos.html }\end{array}$ & $\mathrm{N}$ & $\mathrm{Y}$ & $\mathrm{N}$ & $\mathrm{N}$ & $\mathrm{N}$ & $Y$ & $\mathrm{Y}$ & $\mathrm{N}$ \\
\hline $\begin{array}{l}\text { http://www. freevbc } \\
\text { ode.com/ShowCod } \\
\text { e.asp?ID=4079 }\end{array}$ & $\mathrm{N}$ & $\mathrm{Y}$ & $\mathrm{N}$ & $\mathrm{N}$ & $\mathrm{P}$ & $Y$ & $\mathrm{Y}$ & $\mathrm{N}$ \\
\hline $\begin{array}{l}\text { http://samate.nist.g } \\
\text { ov/index.php/Tool } \\
\text { _Survey.html }\end{array}$ & $\mathrm{N}$ & $\mathrm{Y}$ & $\mathrm{N}$ & $\mathrm{N}$ & $\mathrm{P}$ & $\mathrm{Y}$ & $\mathrm{Y}$ & $\mathrm{N}$ \\
\hline $\begin{array}{l}\text { http://pmd.sourcefo } \\
\text { rge.net/ }\end{array}$ & $\mathrm{N}$ & $\mathrm{Y}$ & $\mathrm{N}$ & $\mathrm{N}$ & $\mathrm{N}$ & $Y$ & $\mathrm{Y}$ & $\mathrm{N}$ \\
\hline $\begin{array}{l}\text { http://www.stachli } \\
\text { u.com/resources/to } \\
\text { ols/google- } \\
\text { hacking-diggity- } \\
\text { project/attack- } \\
\text { tools/ }\end{array}$ & $\mathrm{N}$ & $\mathrm{Y}$ & $\mathrm{Y}$ & $\mathrm{N}$ & $\mathrm{P}$ & $\mathrm{Y}$ & $\mathrm{Y}$ & $\mathrm{N}$ \\
\hline $\begin{array}{l}\text { http://findbugs.sour } \\
\text { ceforge.net/demo.h } \\
\text { tml }\end{array}$ & $\mathrm{N}$ & $\mathrm{Y}$ & $\mathrm{Y}$ & $\mathrm{N}$ & $\mathrm{P}$ & $Y$ & $\mathrm{Y}$ & $\mathrm{P}$ \\
\hline
\end{tabular}




\begin{tabular}{|l|c|c|c|c|c|c|c|c|}
\hline $\begin{array}{l}\text { https://www.fortify } \\
\text {.com/ssa- } \\
\text { elements/threat- } \\
\text { intelligence/rats.ht } \\
\text { ml }\end{array}$ & $\mathrm{N}$ & $\mathrm{Y}$ & $\mathrm{N}$ & $\mathrm{N}$ & $\mathrm{N}$ & $\mathrm{Y}$ & $\mathrm{Y}$ & $\mathrm{N}$ \\
\hline $\begin{array}{l}\text { http://www.models } \\
\text { phere.org/open_mo } \\
\text { delsphere.html }\end{array}$ & $\mathrm{N}$ & $\mathrm{Y}$ & $\mathrm{Y}$ & $\mathrm{N}$ & $\mathrm{N}$ & $\mathrm{Y}$ & $\mathrm{Y}$ & $\mathrm{P}$ \\
\hline $\begin{array}{l}\text { http://www.sqlpow } \\
\text { er.ca/page/architect }\end{array}$ & $\mathrm{N}$ & $\mathrm{Y}$ & $\mathrm{Y}$ & $\mathrm{P}$ & $\mathrm{P}$ & $\mathrm{Y}$ & $\mathrm{Y}$ & $\mathrm{N}$ \\
\hline $\begin{array}{l}\text { http://www.dbvis.c } \\
\text { om/ }\end{array}$ & $\mathrm{N}$ & $\mathrm{Y}$ & $\mathrm{Y}$ & $\mathrm{N}$ & $\mathrm{P}$ & $\mathrm{Y}$ & $\mathrm{Y}$ & $\mathrm{P}$ \\
\hline $\begin{array}{l}\text { http://www.sqlman } \\
\text { ager.net/tools/free }\end{array}$ & $\mathrm{N}$ & $\mathrm{Y}$ & $\mathrm{Y}$ & $\mathrm{N}$ & $\mathrm{P}$ & $\mathrm{Y}$ & $\mathrm{Y}$ & $\mathrm{P}$ \\
\hline http://elphsoft.com/ & $\mathrm{N}$ & $\mathrm{Y}$ & $\mathrm{N}$ & $\mathrm{N}$ & $\mathrm{N}$ & $\mathrm{Y}$ & $\mathrm{Y}$ & $\mathrm{N}$ \\
\hline $\begin{array}{l}\text { http://www.isi.edu/ } \\
\text { nsnam/ns/ }\end{array}$ & $\mathrm{N}$ & $\mathrm{Y}$ & $\mathrm{Y}$ & $\mathrm{N}$ & $\mathrm{N}$ & $\mathrm{Y}$ & $\mathrm{Y}$ & $\mathrm{P}$ \\
\hline $\begin{array}{l}\text { http://www.cc.gate } \\
\text { ch.edu/computing/ } \\
\text { compass/pdns/inde } \\
\text { x.html }\end{array}$ & $\mathrm{N}$ & $\mathrm{Y}$ & $\mathrm{Y}$ & $\mathrm{N}$ & $\mathrm{N}$ & $\mathrm{Y}$ & $\mathrm{Y}$ & $\mathrm{N}$ \\
\hline $\begin{array}{l}\text { http://www.ssfnet. } \\
\text { org/homePage.html }\end{array}$ & $\mathrm{N}$ & $\mathrm{Y}$ & $\mathrm{Y}$ & $\mathrm{N}$ & $\mathrm{N}$ & $\mathrm{Y}$ & $\mathrm{Y}$ & $\mathrm{Y}$ \\
\hline $\begin{array}{l}\text { http://www.cisco.c } \\
\text { om/web/learning/n } \\
\text { etacad/course_catal } \\
\text { og/PacketTracer.ht } \\
\text { ml }\end{array}$ & $\mathrm{P}$ & $\mathrm{Y}$ & $\mathrm{Y}$ & $\mathrm{P}$ & $\mathrm{N}$ & $\mathrm{Y}$ & $\mathrm{Y}$ & $\mathrm{Y}$ \\
\hline $\begin{array}{l}\text { http://www.filebuz } \\
\text { z.com/findsoftware } \\
\text { /Free_Network_Si } \\
\text { mulator/1.html }\end{array}$ & $\mathrm{N}$ & $\mathrm{Y}$ & $\mathrm{P}$ & $\mathrm{N}$ & $\mathrm{N}$ & $\mathrm{Y}$ & $\mathrm{Y}$ & $\mathrm{N}$ \\
\hline $\begin{array}{l}\text { http://www.questio } \\
\text { nwriter.com/ }\end{array}$ & $\mathrm{P}$ & $\mathrm{Y}$ & $\mathrm{Y}$ & $\mathrm{N}$ & $\mathrm{P}$ & $\mathrm{Y}$ & $\mathrm{Y}$ & $\mathrm{N}$ \\
\hline $\begin{array}{l}\text { https://compilr.com } \\
\text { / }\end{array}$ & $\mathrm{Y}$ & $\mathrm{Y}$ & $\mathrm{Y}$ & $\mathrm{N}$ & $\mathrm{Y}$ & $\mathrm{Y}$ & $\mathrm{Y}$ & $\mathrm{Y}$ \\
\hline $\begin{array}{l}\text { http://www.spicyn } \\
\text { odes.org/ }\end{array}$ & $\mathrm{Y}$ & $\mathrm{Y}$ & $\mathrm{Y}$ & $\mathrm{N}$ & $\mathrm{Y}$ & $\mathrm{Y}$ & $\mathrm{Y}$ & $\mathrm{P}$ \\
\hline
\end{tabular}

$Y$ - Yes, Fully Available, $P$ - Partially Available, $N$ No, Not Available

\section{COMPREHENSIVE E-LMS}

Using the knowledge acquired from the survey listed in Table V, a web based e-learning system specific for learning Object Oriented Programming Using $\mathrm{C}++$ was developed. It is a minimal working prototype which gives the look and feel of all the learning components [17] that utilizes various social tools, interactive environments of Web 2.0, various web based services and cloud. The main components of the system are shown in Fig. 2. This proposed system can be further enhanced to accommodate various other topics in future. The proposed system is available at [18]. Some of the key features of the system are as follows:

Cloud is used as a repository for learning objects like presentations, documents, etc. The cloud service used for this purpose is [19]. The service provider provides 500 GB of free storage, freeing the server from the respective overload and enables the researchers to focus on the application.

All the video lessons are uploaded to YouTube.com and played here. This is implemented using the open APIs provided by YouTube.com.

Google Gadgets, like Google Calendar are used to publish the Schedule / Course plan.

Links to different sites for non human resources like animations, references, books, journals, articles and other forms of media are incorporated. On line quizzes produce the result of the quiz and mail it to the stakeholders about the learners' performance. Th is was done using Question writer [20]. A dictionary of all the key words in the $\mathrm{C}++$ language, a custom built software is also provided. The main aim of this elearning web solution is to provide the learner with audio, visual and kinesthetic learning from the tools available in web 2.0. REST API and other API provided by FaceBook are used to build discussion forum and chat room features for those attending the course. (Fig.11). The learners can also test their programs using [21], which provides a platform for program compilation and execution facility for many programming languages. (Fig.8). A question bank is a repository of questions to support the learning process. This web solution also holds a sample question bank for learning $\mathrm{C}^{++}$. (Fig.9). Using [22] mind map is generated to represent and structure ideas and concepts of $\mathrm{C}^{++}$graphically. (Fig.10). Some of the other features included in the system are deriving class diagrams from source code [23], learning by playing cross word puzzle [24], analy zing the code's efficiency using static analyzer [25] and sample $\mathrm{C}^{++}$programs [26].

When the proposed e-LMS was compared with Felder-Silverman model learning styles, the mapping obtained is as listed in Table VI.

TABLE VI: COMPARISON BETWEEN OUR ELEARNING SYSTEM AND FELDER-SILVERMAN MODEL

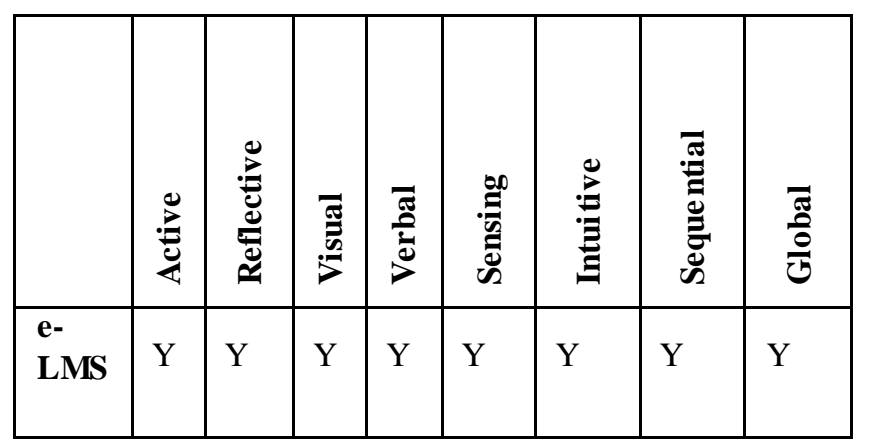




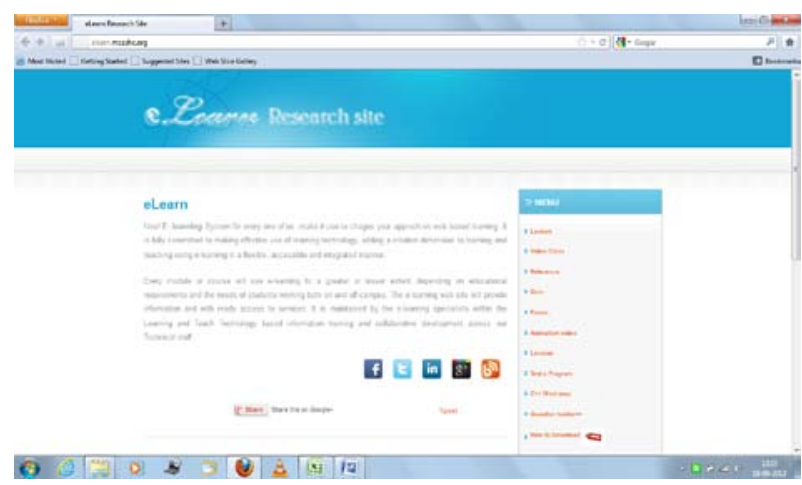

Figure 1. Experimental Web based e-Learning System

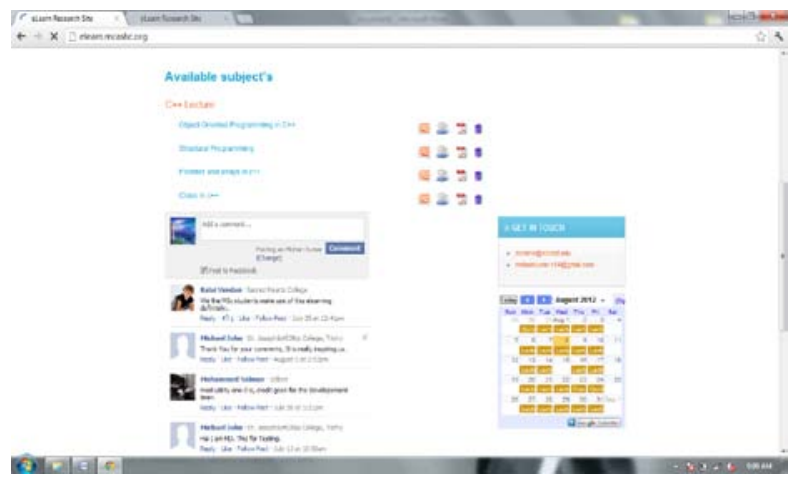

Figure 2.Components of the e-learning system

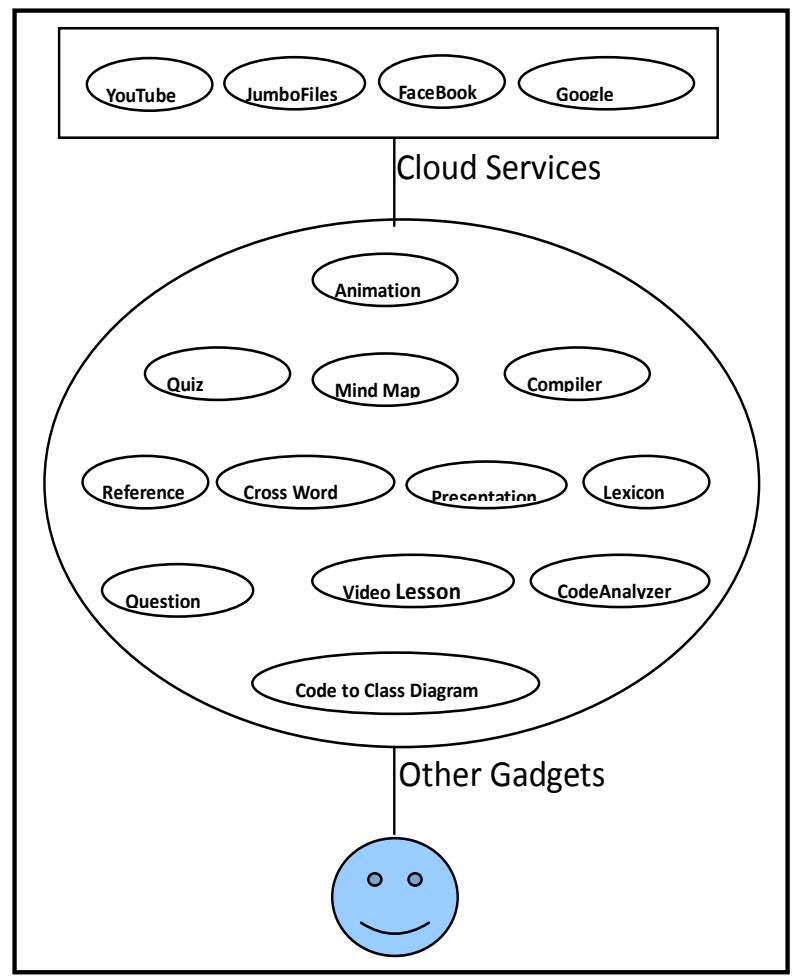

Figure 3. Learning Materials using Jumbo Files

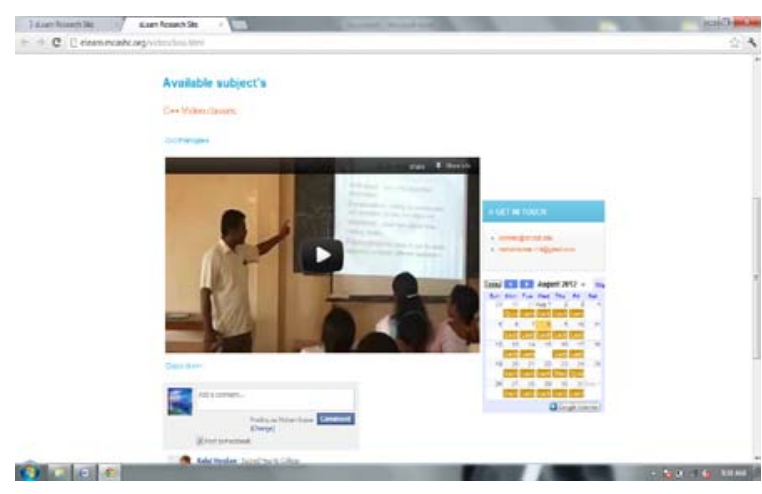

Figure 4. Video Lessons from YouTube

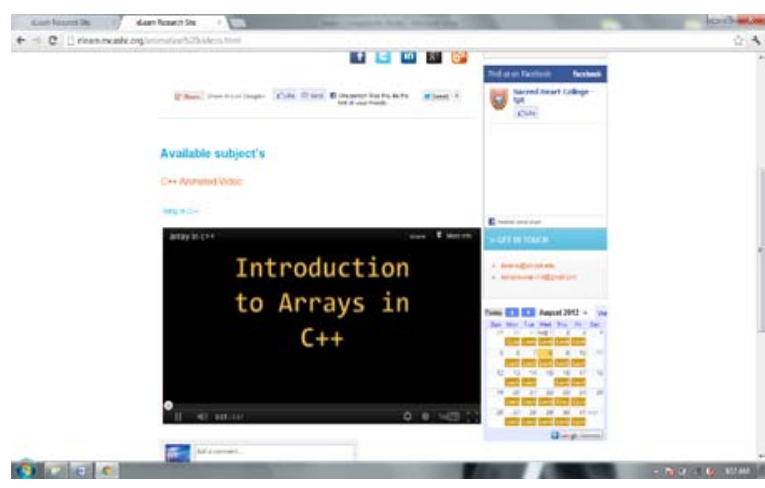

Figure 5. Usage of Animations

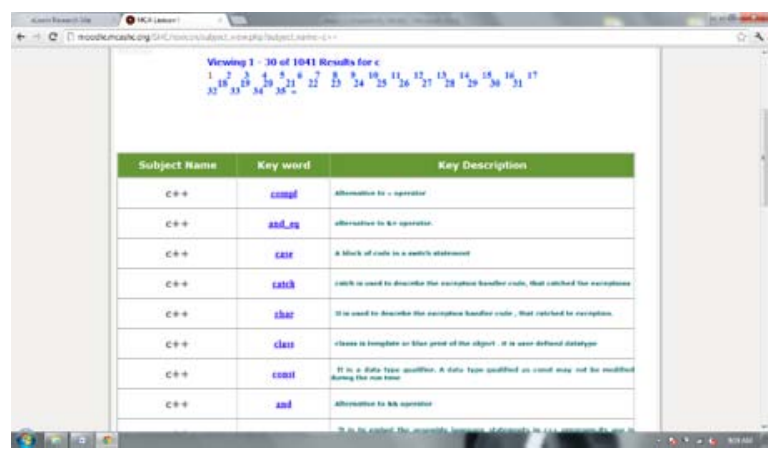

Figure 6. Custom built Lexicon software

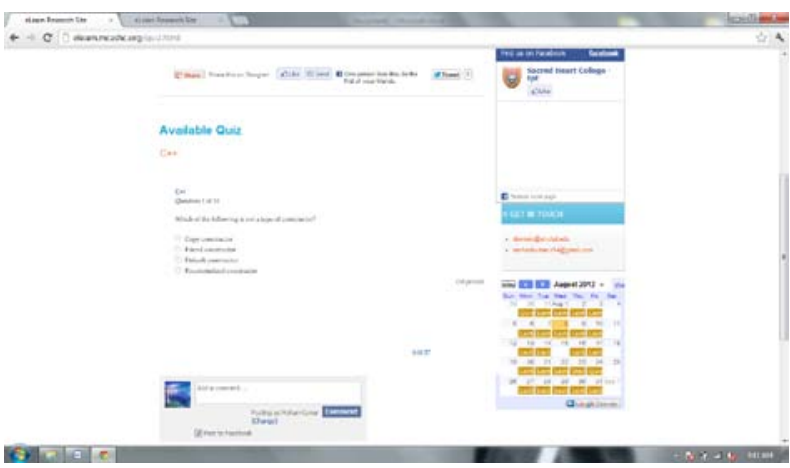

Figure 7. Quiz using questionwriter.com 


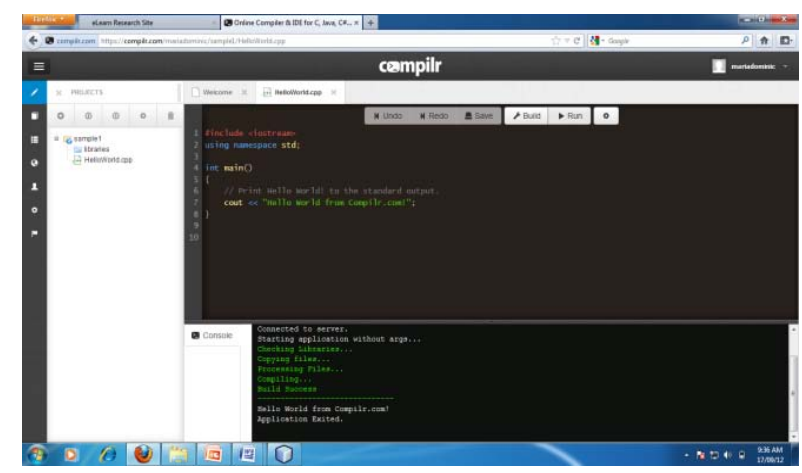

Figure 8. Using compilr.com

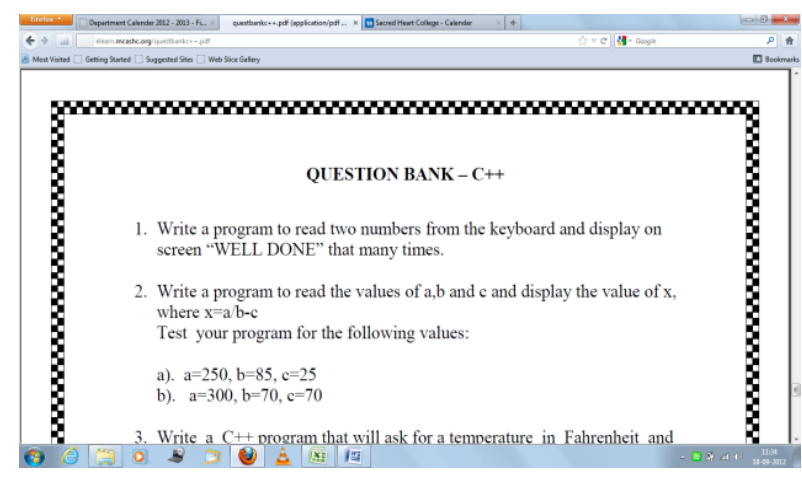

Figure 9. Custom designed Question Bank

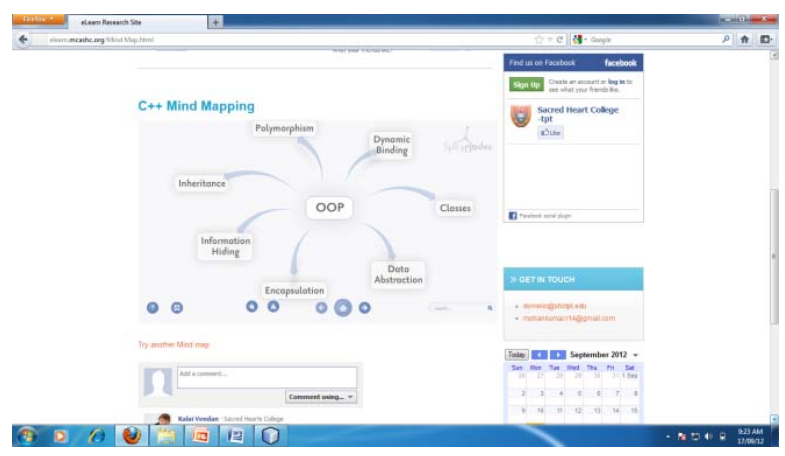

Figure 10. Mind Mapping using spicynodes.com

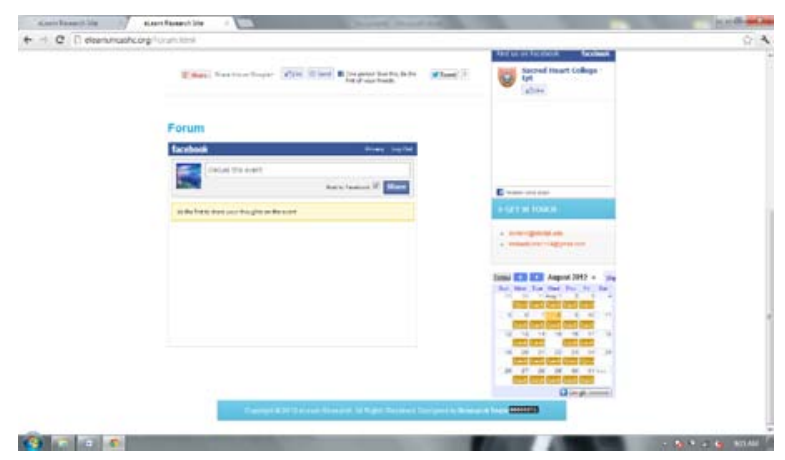

Figure 11. Discussion Forum using FaceBook.com

As seen from Table VI, the experimental research site satisfies all the required characteristics mentioned by the Felder-Silverman model. Similar projects using cloud based e-Learning are Casquero [27] and Virtual Computing Lab [28].

\section{CLUSION}

This study aims to identify e-learning possibilities using social and other tools available in web 2.0. This will enhance the e-learning framework by using the freely available tools to be mashuped together to provide a better e-learning framework according to the need of the stakeholders. Every requirement can have different frameworks. Thus, the above research and the experiment, is bound to motivate and enlighten the stakeholders to go for customized e-learning platforms according to their requirements. Initial feedback from users of this system found it to be interesting and useful than the traditional system. Based on the behavior pattern and other parameters the system needs to become dynamic and adapt according to the learner learning path. This could be the future scope of this system. This can be implemented using the tools available in web 3.0. Another e-LMS for learning Java Programming is also being designed and is available at [29].

\section{ACKNOW LEDGMENT}

The authors wish to thank the management of Sacred Heart College for allowing them to host the experimental website for academic research purpose. The readers are encouraged to give their valuable suggestion in the website.

\section{REFERENCES}

[1] RIDING AND RAYNER, "RECENT ADAPTIVE ELEARNING contributions towards a standard ready architecture”, Innovations in Education and Teaching International, 2006, 43, 121-135.

[2] SOTIRIOS BOTSIOS, DIMITRIOS GEORGIOU, "RECENT ADAPTIVE E-LEARNING CONTRIBUTIONS TOWARDS A “ST ANDARD READY” ARCHITECTURE, INTERNATIONAL JOURNAL OF KNOWLEDGE AND LEARNING, 2009.

[3] DEKSON, SURESH, “ADAPTIVE E-LEARNING TECHNIQUES IN THE DEVELOPMENT OF TEACHING ELECTRONICS PORTFOLIO - A SURVEY”, INTERNATIONAL JOURNAL OF ENGINEERING SCIENCE AND TECHNOLOGY, 2(9), 4175-4181.

[4] HONEY, MUMFORD, KOLB, FELDER AND SILVERMAN,"IN-DEPTH ANALYSIS OF THE FELDERSILVERMAN LEARNING STYLE DIMENSIONS”, 1982, 1984, HTTP://WWW.NCSU.EDU/FELDERPUBLIC/ PAPERS/LS-1988.PDF.

[5] BUTHINA DEEB AND ZAINUDIN BIN HASSAN,"TOWARDS DESIGNING E-LEARNING MATERIALS BASED ON MULTI LEARNER'S ST YLES”, INTERNATIONAL JOURNAL OF COMPUTER APPLICATIONS, 26(3), 2011.

[6] FELDER AND SILVERMAN,"LEARNING AND TEACHING STYLES IN ENGINEERING EDUCATION”, ENGR.EDUCATION, 78(7), 674-681, 1988. 
[7] SARASin, "LEARNING STYLES PERSPECTIVES: IMPACT IN THE CLASSROOM”, MADISON, WI: ATWOOD PUBLISHING.

[8] MONTEMAYOR AND APLATEN, “LEARNing STYLES OF HIGH AND LOW ACADEMIC ACHIEVING FRESHMAN TEACHER EDUCATION STUDENTS: AN APPLICATION OF DUNN AND DUNN'S LEARNING STYLE MODEL” UNIVERSITY OF CORDILLERAS, 1(4): 1-14.

[9] SHAFQAT HAMEED, ATTA BADII, A YESHA PERVAIZ, JOHN MELLOR, ANDREA CULLEN, ”IMPACT OF THE E-LEARNING PACKAGE ON THE QUALTTY OF STUDENT LEARNING EXPERIENCE”, EUROPEAN AND MEDITERRANEAN CONFERENCE ON INFORMATION SYSTEMS, 2009.

[10] POLSANI,"Modalities OF USING LEARNING OBJECTS FOR INTELLIGENT AGENTS IN LEARNING", JOURNAL OF DIGITAL INFORMATION, 3(4), 2003.

[11] BRUSILOVSKY, MELIS, ZHANG,” E-LEARNING AGENTS” INTELLIGENT TUTORING SYSTEMS, BRITISH JOURNAL OF EDUCATIONAL TECHNOLOGY, COMMUNICATIONS OF THE ACM, 2000.

[12] HTTP://EN.WIKIPEDIA.ORG/WIKI/WEB_2.0\#CITE_NO TE- 0 .

[13] CHRISTIAN DALSGAARD,"SOCIAL NETWORKING SITES: TRANSPARENCY IN ONLINE EDUCATION", EUNIS, 2008.

[14] MOHAMmed Al-Zoube, SAMIR Abou El-SEOUd ”, USING SOCIAL NETWORKING SITES AS A PLATFORM FOR E- LEARNING”, ICL, 2009.

[15] DEBRA MARSH, RACHEL PANCKHURST", ELENELEARNING EXCHANGE NETWORKS: REACHING OUT TO BILINGUAL AND MULTICULTURAL UNIVERSITY COLLOBORATION”, HAL, 2007.

[16] Jenny Lagsten, Mathias HatakKa, ”The USE OF ONLINE SOCIAL NETWORKS IN CHINESE COLLOBORATIVE E-LEARNING EDUCATION”, HTTP://ORU.DIVA-PORT AL.ORG / SMASH / GET / DIVA2:424915/FULLTEXT01.

[17] MARIA DOMINIC, SAGAYARAJ FRANCIS, "MAPPING OF E-LEARNING COMPONENTS TO CLOUD COMPUTING”, INTERNATIONAL JOURNAL ON ENGINEERING RESEARCH AND TECHNOLOGY, 2012.

[18] HTTP://WWW.ELEARN.MCASHC.ORG.

[19] WWW.JUMBOFILES.COM.

[20] WWW.QUESTIONWRITER.COM.

[21] WWW.COMPILR.COM.

[22] WWW.SPICYNODES.COM.

[23] HTTP://WWW.UCANCODE.NET/VISUAL_C_MFC_SA MPLES/FREE-C-CPP-CODE-GENERATE-TOOLUML-DRAW.HTM.

[24] HTTP://WWW.SST UTOR.COM/CPP/CWORD2.HTM.

[25] HTTP://CPPCHECK.SOURCEFORGE.NET/.

[26] НTTP://WWW.SYVUM.COM/SQUIZZES/CPP/.

[27] CASQUERO, PORTILlO, RAMON OVELOAR, ROMO AND BENITO," IGOOGLE AND GADGETS AS A PLATFORM FOR INTEGRATING INSTITUTIONAL AND EXETERNAL SERVICES”, MUPPLE'08, PP.37-41, 2008.

[28] HTTP://VCL.NCSU.EDU/.
[29] HTTP://ELEARNINGSHC.HPAGE.CO.IN.

Maria Dominic received his Master's in Computer Science from Bharathidasan University, Trichy, India. He is currently an associate professor in the Department of Computer Applications at Sacred Heart College, Tirupattur, India. He is a research scholar in the field of e-Learning and Cloud Computing.

Dr. Sagayaraj Francis is an associate professor in the Department of Computer Science and Engineering, Pondicherry Engineering College, Pondicherry, India. He specializes in Data Management, Data Modeling, Information Systems and e-Learning. 